\title{
Transfinite Reductions in Orthogonal Term Rewriting Systems
}

(Extended abstract)

\author{
J.R. KENNAWAY 1 , J.W. KLOP 2 , M.R. SLEEP 3 \& F.J. DE VRIES 4 \\ (1) jrk@sys.uea.ac.uk, (2) jwk@cwi.nl, (3) mrs@sys.uea.ac.uk, (4) ferjan@cwi.nl \\ $(2,4)$ CWI, Centre for Mathematics and Computer Science, Amsterdam \\ $(1,3)$ School of Information Systems, University of East Anglia, Norwich
}

\begin{abstract}
Strongly convergent reduction is the fundamental notion of reduction in infinitary orthogonal term rewriting systems (OTRSs). For these we prove the Transfinite Parallel Moves Lemma and the Compressing Lemma. Strongness is necessary as shown by counterexamples. Normal forms, .which we allow to be infinite, are unique, in contrast to $\omega$-normal forms. Strongly converging fair reductions result in normal forms.

In general OTRSs the infinite Church-Rosser Property fails for strongly converging reductions. However for Böhm reduction (as in Lambda Calculus, subterms without head normal forms may be replaced by $\perp$ ) the infinite Church-Rosser property does hold. The infinite Church-Rosser Property for non-unifiable OTRSs follows. The top-terminating OTRSs of Dershowitz c.s. are examples of nonunifiable OTRSs.
\end{abstract}

1985 Mathematics Subject Classification: 68050

1987 CR Categories: F4.1, F4.2

Keywords and Phrases: orthogonal term rewriting systems, infinitary rewriting, strong converging reductions, infinite Church-Rosser Properties, normal forms, Böhm Trees, head normal forms, nonunifiable term rewriting systems.

All authors were partially sponsored by SEMAGRAPH, ESPRIT Basic Research Action 3074. The first author was also partially surported by a SERC Advanced Fellowship, and by SERC grant no. GR/F 91582.

\section{INTRODUCTION}

The theory of Orthogonal Term Rewrite Systems (OTRS) is now well established within theoretical computer science. Comprehensive surveys have appeared recently in [Der90a, Klo91]. In this paper we consider extensions of the established theory to cover infinite terms and infinite reductions.

\subsection{Motivation}

At first sight, the motivation for such extensions might appear of theoretical interest only, with little practical relevance. However, it turns out that both infinite terms and infinite rewriting sequences do have practical relevance.

A practical motivation for studying infinite terms and term rewriting arises in the context of lazy functional languages such as Miranda [Tur85] and Haskell [Hud88]. In such languages it is possible to work with infinite terms, such as the list of all Fibonacci numbers or the list of all primes. This style of programming has been advocated by Turner [Tur85], Peyton-Jones [Pey87] and others. Of course the outcome of a particular computation must be finite, but it is pleasant to define such results as finite portions of an infinite term. It would be even more pleasant to know that nice properties (for example Church-Rosserness) hold for infinite as well 
as finite rewriting, but the standard theory does not tell us this. As we show below, ChurchRosserness is one of several standard results which does not hold for infinite rewriting in general, although it does hold for terms which have an infinite normal form (Theorem 4.1.3).

A second practical motivation for considering infinite reduction sequences arises from the common graph-rewrite based implementations of functional languages. The correspondence between graph rewriting and term rewriting was studied in [Bar87] for acyclic graphs. When cyclic graphs are considered, the correspondence with term rewriting immediately requires consideration of infinite terms and infinite reductions. The correspondence with graphs is the motivation for [Far89].

\subsection{Overview}

With these motivations in mind, we set out to identify precise foundations for transfinite rewriting. A certain amount of care is needed to establish appropriate notions and we do this in Section 2. One can take a topological approach as in [Der89a,b\&90] and consider infinite reduction sequences that are converging to a limit in the metric completion of the space of finite terms. However, converging reductions fail to satisfy some natural properties for orthogonal TRSs. Instead we concentrate on strongly converging reductions as introduced by [Far89], which turn out to be better behaved.

\section{Basic facts for infinitary orthogonal term rewrite systems.}

Transf. Parallel Moves Lemma
Inf. Church-Rosser Property
Unique $\omega$-normal forms
Unique normal forms
Compressing Lemma

Fair reductions result in $\omega$-normal forms [Der90b], (3.4.2.i)

NO (3.1.3)

NO (4.1.1)

NO (4.1.1)

YES (3.3.6)

NO [Far89], (3.2.1) converging reductions

strongly converging reductions

YES (3.1.2)

NO (4.1.1)

NO (4.1.1)

YES (3.3.6)

YES (3.2.5)

partial result in [Far89] normal forms (3.4.2.ii)

\section{(Table 1.1)}

In Section 3 we prove the fundamental results for infinitary orthogonal rewrite systems, as summarized in Table 1.1. Then in Section 4 we show the failure of the infinite Church-Rosser Property for general OTRSs. The counterexample refutes not only the CR-property for strongly converging but also the CR-property for converging reductions studied by Dershowitz c.s. Introducing ideas from Lambda Calculus we eliminate subterms that have no head normal form by reducing them to $\perp$. The new Böhm-reduction $\rightarrow \perp$ has the infinite Church Rosser Property for strongly converging reductions. Normal forms for $\rightarrow \perp$-reduction are so called Böhm Trees: they are unique. Finally we show that orthogonal TRSs in which there are no rule in which a left-hand side of a rule can be unified with the right-hand side have the infinite Church-Rosser Property. This class of orthogonal TRSs includes the top-terminating orthogonal TRSs of Dershowitz c.s. 
The present paper is an extended abstract of a longer paper in preparation by the same authors [Ken90a]. There it will be proved that the infinite Church-Rosserproperty holds for strongly converging reductions in OTRSs that contain at most one collapsing rule, which then has to be of the form $\mathrm{I}(\mathrm{x}) \rightarrow \mathrm{x}$. The full paper will further contain extensions of the theory of needed redexes to infinitary orthogonal term rewriting systems and will unravel the connections between graph rewriting and infinitary term rewriting.

We acknowledge critical reading of an earlier draft by Aart Middeldorp.

\section{INFINITARY ORTHOGONAL TERM REWRITING SYSTEMS}

We briefly recall the definition of a finitary term rewriting system, before we define infinitary orthogonal term rewriting systems involving both finite and infinite terms. For more details the reader is referred to [Der90a] and [Klo91].

\subsection{Finitary term rewriting systems}

A finitary term rewriting system over a signature $\Sigma$ is a pair $(\operatorname{Ter}(\Sigma), R)$ consisting of the set $\operatorname{Ter}(\Sigma)$ of finite terms over the signature $\Sigma$ and a set of rewrite rules $R \subseteq \operatorname{Ter}(\Sigma) \times \operatorname{Ter}(\Sigma)$.

The signature $\Sigma$ consists of a countably infinite set Var $\Sigma$ of variables $(\mathrm{x}, \mathrm{y}, \mathrm{z}, \ldots)$ and a nonempty set of function symbols $(A, B, C, \ldots, F, G, \ldots)$ of various finite arities $\geq 0$. Constants are function symbols with arity 0 . The set $\operatorname{Ter}(\Sigma)$ of finite terms $(t, s, \ldots)$ over $\Sigma$ can be defined as usual: the smallest set containing the variables and closed under function application.

The set $\mathrm{O}(\mathrm{t})$ of occurrences in $\mathrm{t}$ is defined by induction on the structure of $\mathrm{t}$ as follows: $\mathrm{O}(\mathrm{t})$ $=\left\{\langle>\}\right.$ if $t$ is a variable and $O(t)=\left\{\langle>\} \cup\left\{<i, u>\mid 1 \leq i \leq n\right.\right.$ and $\left\langle u>\in O\left(t_{i}\right)\right\}$ if $t$ is of the form $F\left(t_{1}, \ldots, t_{n}\right)$. If $u \in O(t)$ then the subterm $t / u$ at occurrence $u$ is defined by induction: $t /\langle>=t$ and $\left.\mathrm{F}\left(\mathrm{t}_{1}, \ldots, \mathrm{t}_{\mathrm{n}}\right) /<\mathrm{i}, \mathrm{u}\right\rangle=\mathrm{t}_{\mathrm{j}} / \mathrm{u}$. The depth of a subterm of $\mathrm{t}$ at $\mathrm{u}$ is the length of $\mathrm{u}$.

Contexts are terms in $\operatorname{Ter}(\Sigma \cup\{\square\})$, in which the special constant $\square$, denoting an empty place, occurs exactly once. Contexts are denoted by $\mathrm{C}[\mathrm{]}$ and the result of substituting a term $\mathrm{t}$ in place of $\square$ is $C[t] \in \operatorname{Ter}(\Sigma)$. A proper context is a context not equal to $\square$.

Substitutions are maps $\sigma: \operatorname{Var}_{\Sigma} \rightarrow \operatorname{Ter}(\Sigma)$ satisfying $\sigma\left(F\left(t_{1}, \ldots, t_{n}\right)\right)=F\left(\sigma\left(t_{1}\right), \ldots, \sigma\left(t_{n}\right)\right)$.

The set $\mathrm{R}$ of rewrite rules contains pairs $(\mathrm{l}, \mathrm{r})$ of terms in $\operatorname{Ter}(\Sigma)$, written as $\mathrm{l} \rightarrow \mathrm{r}$, such that the left-hand side $l$ is not a variable and the variables of the right-hand side $\mathrm{r}$ are contained in 1 . The result $1 \sigma$ of the application of the substitution of $\sigma$ to the term $l$ is called an instance of 1 . A redex (reducible expression) is an instance of a left-hand side of a rewrite rule. A reduction step $t \rightarrow s$ is a pair of terms of the form $C\left[l^{\sigma}\right] \rightarrow C\left[r^{\sigma}\right]$, where $1 \rightarrow r$ is a rewrite rule in $R$. Concatenating reduction steps we get a finite reduction sequence $t_{0} \rightarrow t_{1} \rightarrow \ldots \rightarrow t_{n}$, which we also denote by $t_{0} \rightarrow_{n} t_{n}$, or an infinite reduction sequence $t_{0} \rightarrow t_{1} \rightarrow \ldots$.

\subsection{Infinitary orthogonal term rewriting systems}

An infinitary term rewriting system over a signature $\Sigma$ is a pair $\left(\operatorname{Ter}^{+\infty}(\Sigma), \mathrm{R}\right)$ consisting of the set $\operatorname{Ter}^{\infty}(\Sigma)$ of finite and infinite terms over the signature $\Sigma$ and a set of rewrite rules $\operatorname{R} \subseteq \operatorname{Ter}(\Sigma) \times \operatorname{Ter}^{\infty}(\Sigma)$. We don't consider rewrite rules with infinite left-hand sides, but righthand sides may be infinite in order to interpret various liberal forms of graph rewriting in infinitary term rewriting. In [Der90b] only finite left- and right-sides are considered. 
It takes some elaboration to define the set $\operatorname{Te}^{\circ}(\Sigma)$ of finite and infinite terms. Finite terms may be represented as finite trees, well-labelled with variables and function symbols. Welllabelled means that a node with $n \geq 1$ successors is labelled with a function symbol of arity $n$ and that a node with no successors is labelled either with a constant or a variable. Now infinite terms are infinite well-labelled trees with nodes at finite distance to the root. Substitutions, contexts and reduction steps generalize trivially to the set of infinitary terms $\operatorname{Ter}^{\infty}(\Sigma)$.

To introduce the prefix ordering $\leq$ on terms we extend the signature $\Sigma$ with a fresh symbol $\Omega$. The prefix ordering $\leq$ on $\operatorname{Ter}^{\infty}(\Sigma \cup\{\Omega\})$ is defined inductively: $x \leq x$ for any variable $x, \Omega \leq$ $t$ for any term $t$ and if $t_{1} \leq s_{1}, \ldots, t_{n} \leq s_{n}$ then $F\left(t_{1}, \ldots, t_{n}\right) \leq F\left(s_{1}, \ldots, s_{n}\right)$.

If all function symbols of $\Sigma$ occur in $R$ we will write just $R$ for $\left(\operatorname{Ter}^{\infty}(\Sigma), R\right)$. The usual properties for finitary TRSs extend verbatim to infinitary TRSs:

\subsubsection{DEFINITION. Let $R$ be an infinitary TRS.}

(i) $\mathrm{R}$ is left-linear if no variable occurs more than once in a left-hand side of $\mathrm{R}$ 's rewrite rules;

(ii) (informally) $\mathrm{R}$ is non-overlapping (or non-ambiguous) if non-variable parts of different rewrite rules don't overlap and non-variable parts of the same rewrite rule overlap only entirely:

(ii') (formally) $\mathrm{R}$ is non-overlapping if for any two left-hand sides $\mathrm{s}$ and $\mathrm{t}$, any occurrence $\mathrm{u}$ in $t$, and any substitutions $\sigma$ and $\tau: \operatorname{Var}_{\Sigma} \rightarrow \operatorname{Ter}(\Sigma)$ it holds that if $(t / u)^{\sigma}=s^{\tau}$ then either $t / u$ is a variable or $t$ and $s$ are left-hand sides of the same rewrite rule and $u$ is the empty occurrence $<>$, the occurrence of the root.

(iii) $\mathrm{R}$ is orthogonal if $\mathrm{R}$ is both left-linear and non-overlapping.

It is well-known (cf. [Ros73], [Klo91]) that finitary orthogonal TRSs satisfy the finitary Church-Rosser property, i.e., ${ }^{*} \leftarrow \bullet \rightarrow^{*} \subseteq \rightarrow^{*}{ }^{*} \leftarrow$, where $\rightarrow^{*}$ is the transitive, reflexive closure of the relation $\rightarrow$. It is obvious that infinitary orthogonal TRSs inherit this finitary property.

In the present infinitary context it is natural to define that a term is a normal form if it contains no redexes, just like in the finitary context. A term $t$ has a normal form $s$ if there is a reduction $t$ $\rightarrow_{\alpha}$ s. Dershowitz, Kaplan and Plaisted [Der89a, Der89b and Der90b] consider a weaker, more liberal notion of normal form: the $\omega$-normal forms. An $\omega$-normal form is a term such that if this term can reduce, then it reduces in one step to itself. One sees easily that restricted to finite terms normal forms and $\omega$-normal forms are already different concepts: in the TRS with rule $A \rightarrow A$ the term $\mathrm{A}$ is an $\omega$-normal form, but not a normal form.

\subsection{Converging and strongly converging transfinite reductions}

Generalizing the finite situation we would like to express that there is a reduction of length $\alpha+1$ that transforms to into $t_{\alpha}$, where $\alpha$ may be any ordinal. Compare the following three reductions of length $\omega$, the corresponding TRSs are easy to imagine:

(i) $\mathrm{A} \rightarrow \mathrm{B} \rightarrow \mathrm{A} \rightarrow \mathrm{B} \rightarrow \ldots$,

(ii) $\mathrm{C} \rightarrow \mathrm{S}(\mathrm{C}) \rightarrow \mathrm{S}(\mathrm{S}(\mathrm{C})) \rightarrow \ldots$,

(iii) $\mathrm{D}(\mathrm{E}) \rightarrow \mathrm{D}(\mathrm{S}(\mathrm{E})) \rightarrow \mathrm{D}(\mathrm{S}(\mathrm{S}(\mathrm{E}))) \rightarrow \ldots$.

Clearly in the first reduction $A$ will not be transformed in the limit to anything fixed, in contrast to $C$ and $D(E)$ in the second and third reduction. It is tempting to say that the limit of $C$ will be $S \omega$, an infinite reduction of $S$ (plus all the necessary brackets), and similar $D(E)$ should have as limit $D\left(S^{\omega}\right)$. Cauchy convergence is the natural formalism in which to express all this. 
The set $\operatorname{Ter}(\Sigma)$ of finite terms for a signature $\Sigma$ can be provided with an ultra-metric d: $\operatorname{Ter}(\Sigma) \times \operatorname{Ter}(\Sigma) \rightarrow[0,1]$ (cf. e.g. [Arn80]). The distance $d(t, s)$ of two terms $t$ and $s$ is 0 if $t$ and $s$ are equal, and otherwise $2^{-k}$, where $k \in \mathbb{N}$ is the largest number such that the labels of all nodes of $s$ and $t$ at depth less than or equal to $k$ are equally labelled. The metric completion of $\operatorname{Ter}(\Sigma)$ is isomorphic to the set of infinitary terms $\operatorname{Ter}^{\infty}(\Sigma)$ (cf. [Arn80])

In the complete metric space $\operatorname{Ter}^{\infty}(\Sigma)$ all Cauchy sequences of ordinal length $\alpha$ have a limit. We will now recall the transfinite converging reductions by Dershowitz, Kaplan and Plaisted [Der90b].

2.3.1. DEFINITION. A sequence of length $\alpha$ is a set of elements indexed by some ordinal $\alpha \geq$ 1: notation $\left(t_{\beta}\right) \beta<\alpha$. Instead of $\left(t_{\beta}\right) \beta<\alpha+1$ we often write $\left(t_{\beta}\right) \beta \leq \alpha$.

2.3.2. Definition. By induction on the ordinal $\alpha$ we define when a sequence $\left(t_{\beta}\right) \beta \leq \alpha$ is a converging sequence towards its limit $t_{\alpha}$ (notation: $t_{0} \rightarrow_{\alpha}^{c} t_{\alpha}$ ):

(i) $t_{0} \rightarrow{ }_{0}^{c} t_{0}$,

(ii) $t_{0} \rightarrow{ }_{\beta+1}^{c} t_{\beta+1}$ if $t_{0} \rightarrow_{\beta}^{c} t_{\beta}$,

(iii) $\mathrm{t}_{0} \rightarrow{ }_{\lambda}^{\mathrm{c}} \mathrm{t} \lambda$ if $\mathrm{t}_{0} \rightarrow{ }_{\beta}^{\mathrm{c}} \mathrm{t}_{\beta}$ for all $\beta<\lambda$ and $\forall \varepsilon>0 \exists \beta<\lambda \forall \gamma\left(\beta<\gamma<\lambda \rightarrow \mathrm{d}\left(\mathrm{t}_{\gamma}, \mathrm{t}_{\lambda}\right)<\varepsilon\right)$.

This definition of transfinite convergence is an instance of the so-called Moore-Smith convergence over nets (cf. for instance [Kel55]). Limits are unique: if the topological space is a Hausdorff space then each net in the space converges to at most one point; the spaces $\operatorname{Ter}(\Sigma)$ and $\operatorname{Ter}^{\infty}(\Sigma)$ are Hausdorff spaces.

2.3.3. DEFINITION. A reduction of length $\alpha \geq 1$ is a sequence $\left(t_{\beta}\right)_{\beta<\alpha}$ such that $t_{\beta} \rightarrow t_{\beta+1}$ for all $\beta$ such that $\beta+1<\alpha$. The redex contracted by the step $t_{\beta} \rightarrow t_{\beta+1}$ will be denoted by $R_{\beta}$, its depth as subterm of $t_{\beta}$ by $d \beta$.

We will now define strong reductions as reductions in which the depth of the reduced redexes tends to infinity. We present the definition for reductions of arbitrary transfinite length.

2.3.4. DEFINITION. By induction on the ordinal $\alpha \geq 1$ we define when a reduction $(\mathrm{t} \beta)_{\beta<\alpha}$ is a strong reduction:

(i) $\left(t_{\beta}\right) \beta<1$ is a strong reduction;

(ii) $\left(t_{\gamma}\right)_{\gamma<\beta+1}$ is a strong reduction if $\left(t_{\gamma}\right)_{\gamma<\beta}$ is a strong reduction;

(iii) $\left(t_{\gamma}\right)_{\gamma<\lambda}$ is a strong reduction if for all $\beta<\lambda$ the reduction $\left(t_{\gamma}\right)_{\gamma<\beta}$ is strong and $\forall \mathrm{d}>0 \exists \beta<\lambda \forall \gamma\left(\beta \leq \gamma<\lambda \rightarrow \mathrm{d}_{\gamma}>\mathrm{d}\right)$.

2.3.5. DEFINITION. A strongly converging reduction is a converging sequence that is a strong reduction.

The strongly converging reductions are of importance for the theory of infinitary term rewriting. Therefore we denote a strongly converging reduction $\left(t_{\beta}\right)_{\beta \leq \alpha}$ by $t_{0} \rightarrow_{\alpha} t_{\alpha}$ By $t \rightarrow \leq \alpha s$ we denote the existence of a strong reduction of length less than or equal to $\alpha$ converging towards limit $\mathrm{s}$. We use a similar notation $t \rightarrow_{\leq \alpha}^{c} \mathrm{~s}$ for converging reductions of length less than or equal to $\alpha$. 
The second example of this section is an example of a strongly converging reduction. Other examples of strongly converging reductions are found in (3.2.1.ii) and (4.1.1).

\subsection{Counting steps in strongly converging reductions}

Convergent transfinite reductions exist of any length. Consider for example the TRS with the single rule $\mathrm{A} \rightarrow \mathrm{A}$. Reductions of the form $\mathrm{A} \rightarrow{ }_{\alpha}^{\mathrm{C}} \mathrm{A}$ are converging for any ordinal $\alpha$. However these sequences are not strongly convergent. The example $A \rightarrow_{\alpha} A$ shows also that in a converging reduction any number of reduction steps may be performed below some depth. For strongly converging reductions this is different:

2.4.1. THEOREM. If $t_{0} \rightarrow \lambda t_{\lambda}$ is strongly convergent, then the number of steps in $t_{0} \rightarrow \lambda t_{\lambda}$ reducing a redex at depth $\leq \mathrm{n}$ is finite.

PROOF. Assume $t_{0} \rightarrow \lambda t_{\lambda}$ is strongly convergent. As this reduction is strong there is a last step $t_{\alpha} \rightarrow t_{\alpha+1}$ at which a redex is contracted at depth $\leq n$. Consider the initial segment $t_{0} \rightarrow \alpha t_{\alpha}$, and repeat the argument. By the well-ordering of the ordinals (no infinite descending chains of ordinals) this process stops in finitely many steps.

2.4.2. COROLLARY. A strongly converging transfinite reduction has countable length.

PROOF. By the previous Theorem 2.4.1 a strongly convergent transfinite reduction can only perform finitely many reductions at any given depth $d \in \mathbb{N}$.

\section{FUNDAMENTAL FACTS OF INFINITARY TERM REWRITING}

From now on we consider infinitary orthogonal term rewriting systems, except in 3.4.

\subsection{The Transfinite Parallel Moves Lemma}

In $t \rightarrow s$ let $s$ be obtained by contraction of the redex $S$ in $t$. Recall the notation ulS of the set descendants of a redex occurrence $u$ of $t$ in the contraction of $S$ (cf. [Hue79]). Descendance can be extended to transfinite reductions:

3.1.1. DEFINITION. Let $t_{0} \rightarrow \alpha t_{\alpha}$ be a transfinite strongly converging reduction such that for all $\beta<\alpha t_{\beta}$ reduces to $t_{\beta+1}$ by contraction of the redex $R_{\beta}$. By induction on the ordinal $\alpha$ we define the set of descendants $u \backslash \alpha$ in $t_{\alpha}$ that descend from the redex occurrence $u$ in $t_{0}$ :

(i) $\mathrm{u} \mathrm{WO}=\{\mathrm{u}\}$

(ii) $u \backslash(\beta+1)=\bigcup\left\{v \backslash R_{\beta} \mid v \in u \backslash \beta\right\}$

(iii) $u h=\{v \mid \exists \beta<\lambda \forall \gamma(\beta \leq \gamma<\lambda \rightarrow v \in u \backslash \gamma)\}$

\subsubsection{TRANSFINITE PARALLEL MOVES LEMMA.}

Let $t_{0} \rightarrow \alpha t_{\alpha}$ be a strongly converging reduction sequence of $t_{0}$ with limit $t_{\alpha}$ and let $t_{0} \rightarrow s_{0}$ be a reduction of a redex $S$ of $t_{0}$. Then for each $\beta \leq \alpha$ a term $s \beta$ can be constructed by outermost contraction of all descendants of $S$ in $t_{\beta}$ such that $\mathrm{s}_{\beta} \rightarrow{ }^{*} \mathrm{~s}_{\beta+1}$ for each $\beta \leq \alpha$ and all these reductions together form a strongly converging reduction from $\mathrm{s}_{0}$ to $\mathrm{s}_{\alpha}$. (See Figure 3.1) 


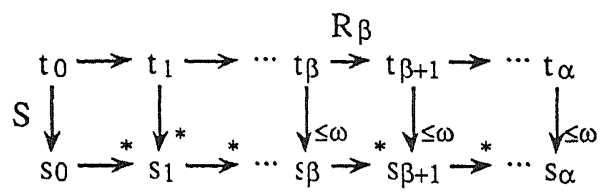

(Figure 3.1)

PROOF. First note that outermost reduction of a finite or an infinite number of disjoint redexes in some term gives a strongly converging reduction. hence all vertical reductions in Figure 3.1 are strongly converging.

We prove the lemma by induction on the ordinal $\alpha$. The case with zero is easy. Next, let $\alpha$ be of the form $\beta+1$. This goes like the traditional proof, taking care of the possible infinite righthand sides. Finally, let $\alpha$ be a limit ordinal $\lambda$. Assume as induction hypothesis that we have the Transfinite Parallel Moves Lemma for $\beta<\lambda$. There are two possibilities: there exists a $\beta<\lambda$ such that the actual length of the reduction sequence $t_{\beta} \rightarrow \leq \omega s \beta$ is zero, that is there are no descendants of $S$ in $t_{\beta}$, or there is no such $\beta$. The first possibility is easy: we find that $t_{\gamma}=s_{\gamma}$ for all $\gamma$ with $\beta \leq \gamma<\lambda$. It follows that so strongly converges to $s \lambda$.

So let us pursue the second possibility and suppose there is no such $\beta$.

Let $\left(v_{\beta}\right)_{\beta \leq \mu}$ be the reduction of the bottom line of Figure 3.1 obtained by refining the sequence $\left(s_{\beta}\right)_{\beta \leq \lambda}$ with reductions $s \beta \rightarrow \leq \omega s \beta+1$ for each $\beta<\alpha$. That such a $\mu$ exists follows by an exercise on well-orderings: refining a well-ordering with well-orderings gives again a wellordering. In order to conclude $s_{0}=v_{0} \rightarrow_{\mu} v_{\mu}=s_{\lambda}$ we have to show: (i) the reduction ( $\left.v_{\beta}\right)_{\beta} \mu \mu$ is strong, (ii) the reduction $\left(v_{\beta}\right)_{\beta} \leq \mu$ is converging. But this is straigthforward.

It seems natural to ask whether a transfinite parallel moves lemma exists for the larger class of converging reductions. The following example shows that the construction embodied in the Transfinite Parallel Moves Lemma for strongly converging reductions does not generalize.

\subsubsection{COUNTEREXAMPLE.}

Rules: $\quad \mathrm{A}(\mathrm{x}, \mathrm{y}) \rightarrow \mathrm{A}(\mathrm{y}, \mathrm{x}), \mathrm{C} \rightarrow \mathrm{D}$

Sequences: $\quad \mathrm{A}(\mathrm{C}, \mathrm{C}) \rightarrow \underset{\downarrow}{\mathrm{A}(\mathrm{C}, \mathrm{C})} \rightarrow \underset{\downarrow}{\mathrm{A}(\mathrm{C}, \mathrm{C})} \rightarrow \underset{\downarrow}{\mathrm{A}(\mathrm{C}, \mathrm{C})} \rightarrow \ldots \rightarrow \underset{\omega}{\mathrm{c}} \rightarrow \underset{\not}{\mathrm{A}(\mathrm{C}, \mathrm{C})}$

$$
\mathrm{A}(\mathrm{C}, \mathrm{D}) \rightarrow \mathrm{A}(\mathrm{D}, \mathrm{C}) \rightarrow \mathrm{A}(\mathrm{C}, \mathrm{D}) \rightarrow \mathrm{A}(\mathrm{D}, \mathrm{C}) \rightarrow \ldots \quad \text { NO LIMIT }
$$

The bottom infinite reduction obtained by standard projection over the one step reduction $\mathrm{C} \rightarrow \mathrm{D}$ does not converge to any limit.

Note that this example is a counterexample not to the Parallel Moves Lemma, but to a method of proving it. It might be possible that by altering the construction, perhaps by considering a more liberal notion of descendant, the parallel moves lemma holds for transfinite converging reductions. After all, every term occurring in the counterexample can reduce to $A(D, D)$.

\subsection{The Compressing Lemma}

In this section we will prove the Compressing Lemma for infinitary left-linear TRSs: if $\mathrm{t} \rightarrow_{\alpha} \mathrm{s}$ is strongly converging, then $t \rightarrow \leq \omega$. That is: any strongly converging reduction from $t$ into $s$ of 
length $\alpha+1$ can be compressed in a reduction of length lesser or equal than $\omega+1$. The conditions left-linearity and strongly converging are necessary:

3.2.1. COUNTEREXAMPLES.

(i) Example against a compressing lemma for converging reductions in orthogonal TRSs.

Rules: $\mathrm{A}(\mathrm{x}) \rightarrow \mathrm{A}(\mathrm{B}(\mathrm{x})), \mathrm{B}(\mathrm{x}) \rightarrow \mathrm{E}(\mathrm{x})$

Sequence: $A(C) \rightarrow{ }_{\omega} A\left(B\left(B^{\omega}\right)\right) \rightarrow A\left(E\left(B^{\omega}\right)\right)$.

Note: $A(C)$ cannot reduce to $A\left(E\left(B^{\omega}\right)\right)$ in $\leq \omega$ steps. The reduction is converging but not strong.

(ii) Example of [Der89a] against a compressing lemma for strongly converging reductions in non-left-linear, non-overlapping TRSs.

Rules: $A \rightarrow S(A), B \rightarrow S(B), H(x, x) \rightarrow C$

Sequence: $\mathrm{H}(\mathrm{A}, \mathrm{B}) \rightarrow^{*} \mathrm{H}(\mathrm{S}(\mathrm{A}), \mathrm{S}(\mathrm{B})) \rightarrow^{*} \mathrm{H}(\mathrm{S}(\mathrm{S}(\mathrm{A})), \mathrm{S}(\mathrm{S}(\mathrm{B}))) \rightarrow \rightarrow_{\omega} \mathrm{H}\left(\mathrm{S}^{\omega}, \mathrm{S}^{\omega}\right) \rightarrow \mathrm{C}$

Note: The term $H(A, B)$ of Dershowitz and Kaplan (cf. [Der89a]) can reduce via the limit $\mathrm{H}\left(\mathrm{S}^{\omega}, \mathrm{S}^{\omega}\right)$ to $\mathrm{C}$. But not $\mathrm{H}(\mathrm{A}, \mathrm{B}) \rightarrow \leq \omega \mathrm{C}$. The sequence is strongly converging.

The proof of the Compressing Lemma will go in two steps whose proofs we skip.

3.2.2. COMPRESSING LEMMA for $\omega+1$. If $\mathrm{t} \rightarrow \omega+1 \mathrm{~s}$ is strongly converging, then $\mathrm{t} \rightarrow \leq \omega \mathrm{s}$.

3.2.4. COMPRESSING LEMMA for limit ordinals. If $t_{0} \rightarrow_{\lambda} t_{\lambda}$ is strongly convergent, then there exists a strongly convergent reduction $t_{0} \rightarrow \leq \omega t \lambda$.

3.2.5. GENERAL COMPRESSING LEMMA. For any ordinal $\alpha$ if $\mathrm{t} \rightarrow \alpha \mathrm{t}_{\alpha}$ is strongly convergent, then there exists a strongly convergent reduction $t \rightarrow \leq \omega$ t $\alpha$.

PROOF. Together 3.2.4 and 3.2.2 establish the Compressing Lemma. Every infinite ordinal $\alpha$ has the form $\lambda+n$, for a limit ordinal $\lambda$ and a finite $n$. For any strongly convergent sequence $t \rightarrow \lambda_{+n} t_{\alpha}$, we apply Theorem 3.3.4 to the first $\lambda$ steps, to obtain a sequence $t \rightarrow \leq \omega+n t_{\alpha}$, then apply Theorem 3.2.2 $\mathrm{n}$ times to obtain $\mathrm{t} \rightarrow \leq \omega \mathrm{t} \alpha$.

\subsection{The unique normal form property}

We will show for infinitary orthogonal TRSs that each term has at most one normal form. In contrast, Example 4.1.1 shows that the unique $\omega$-normal form property does not hold in general. To obtain the positive result we need the notion of a stable reduction. Informally, an infinite reduction is stable if the sequence of stable prefixes of its terms converges to its limit: a stable prefix of a term $t$ is a prefix of $t$ such that no occurrence of that prefix can become an occurrence of a redex in any strongly converging reduction starting from $t$. Stable reductions will be strongly converging.

3.3.1. DEFINITION. (i) A prefix $s \leq t$ is stable with respect to a reduction if no occurrence of $s$ becomes an occurrence of a redex during that reduction.

(ii) A prefix $s \leq t$ is stable if $s$ is stable for all possible strongly converging reduction sequences from $t$.

The restriction in part (ii) to strong reductions is technically convenient. For terms having a normal form, it is in fact unnecesssary; the following proposition may be proved by use of the Transfinite Parallel Moves Lemma. We omit the proof. 
3.3.2. PROPOSITTON. In an orthogonal TRS: If a prefix $\mathrm{t}$ of $\mathrm{t}_{0}$ is stable with respect to a strong reduction from to which converges to normal form, then it is stable.

3.3.3. DEFINITION. Let $\Sigma(t)$ denote the maximal stable prefix of t. A converging reduction $\mathrm{t}_{0} \rightarrow \leq \omega \mathrm{t} \omega$ is called stable if $\forall \mathrm{d} \exists N \forall \mathrm{k} \geq \mathrm{N}\left|\Sigma\left(\mathrm{t}_{\mathrm{k}}\right)\right|>\mathrm{d}$, where $|\mathrm{t}|$ denotes the minimal distance of an occurrence of $\Omega$ in $t$ to the root, if there is any, otherwise $|t|=\infty$.

Stability is a very strong condition. The limit of an infinite stable reduction sequence is a normal form, from which it easily follows that stable reduction is Church-Rosser. The proof of the following lemma is routine and therefore omitted.

3.3.4. LEMMA. (i) If $\mathrm{t} \rightarrow \mathrm{s}$ then $\Sigma(\mathrm{t}) \leq \Sigma(\mathrm{s})$.

(ii) For reductions: stable $\Rightarrow$ strongly convergent $\Rightarrow$ convergent. But not conversely.

(iii) The limit of a stable reduction sequence is a normal form.

\subsubsection{THEOREM. The following are equivalent:}

(i) $\mathrm{t} \rightarrow \leq \omega \mathrm{s}$ is a converging reduction to normal form;

(ii) $\mathrm{t} \rightarrow \leq \omega \mathrm{s}$ is a strong converging reduction to normal form;

(iii) $\mathrm{t} \rightarrow \leq \omega \mathrm{s}$ is a stable reduction

Some comments on the proof: It is trivial to see that (iii) $\Rightarrow$ (ii) $\Rightarrow$ (i). The proof of (i) $\Rightarrow$ (ii) is a reductio ad absurdum. The proof of (ii) $\Rightarrow$ (iii) has become easy by Proposition 3.3.2.

\subsubsection{UNIQUE NORMAL FORM PROPERTY. Normal forms are unique in orthogonal TRSs.}

PROOF. Suppose a term $\mathrm{t}$ admits two converging reductions $\mathrm{t} \rightarrow \mathrm{s}_{1} \rightarrow \mathrm{s}_{2} \rightarrow \ldots \rightarrow{ }_{\mathrm{s}}^{\mathrm{c}} \mathrm{s}$ and $\mathrm{t} \rightarrow$ $\mathrm{r}_{1} \rightarrow \mathrm{r}_{2} \rightarrow \ldots \rightarrow_{\leq \omega}^{\mathrm{c}} \mathrm{r}$ to normal form. By Theorem 3.3.5 these reductions are stable. By the finite Church-Rosser property, for each $n$ there exists $u_{n}$ such that $s_{n} \rightarrow^{*} u_{n}$ and $r_{n} \rightarrow^{*} u_{n}$. We obtain $\mathrm{t} \rightarrow^{*} \mathrm{u}_{1} \rightarrow^{*} \mathrm{u}_{2} \rightarrow^{*} \ldots$. Using Lemma 3.3.4 (i) the newly constructed reduction $\left(\mathrm{u}_{\mathrm{n}}\right)_{\mathrm{n} \in \mathbb{N}}$ inherits its stableness from the stable reductions $\left(s_{n}\right)_{n \in \mathbb{N}}$ and $\left(r_{n}\right)_{n \in \mathbb{N}}$. Thus we see by Theorem 3.3.5 that the limit $u$ of $\left(u_{n}\right)$ is a normal form. By Lemma 3.3.4 (i) we see that $\Sigma\left(s_{n}\right) \leq \Sigma\left(u_{n}\right)$ and $\Sigma\left(r_{n}\right) \leq \Sigma\left(u_{n}\right)$. Hence $s \equiv \lim \Sigma\left(s_{n}\right) \leq \lim \Sigma\left(u_{n}\right) \equiv u \geq \lim \Sigma\left(r_{n}\right) \equiv$ r. Since normal forms are maximal in the prefix ordering (in contrast to $\omega$-normal forms) $s$ and $r$ are equal.

It is not difficult to show that any normal form that can be reached via a converging reduction, might also be reached via a strongly converging reduction.

\subsection{Fair reductions}

Theorem 3.3.5 implies that stable converging reductions result in normal forms. If we add a fairness condition to strongly converging reductions, then their limits will also be normal forms. The same fairness condition added to converging reductions results in converging reductions to $\omega$-normal form [Der89b]. Fairness of a reduction will express that, whenever a redex occurs in a term during this reduction, the redex itself or a term containing the redex will be reduced within a finite number of steps.

3.4.1. DEFINITION. (i) Let $r$ be a redex of $t$ at occurrence $u$. A reduction $t \rightarrow_{\leq \omega} t^{\prime}$ preserves $r$ if no step of this reduction performs a contraction at an occurrence $\leq u$. 
(ii) A reduction $t \rightarrow \leq_{\omega} t^{\prime}$ is fair if for every term $t$ " in the reduction, and every redex $r$ of $t$ " some finite part of this reduction starting at $t^{\prime \prime}$ does not preserve $r$.

Note that a finite sequence is fair if and only if it ends in a normal form, and fair reductions don't need to be converging. Note also that orthogonality guarantees that if the reduction $t \rightarrow \leq \omega$ $t^{\prime}$ preserves a redex in $t$ of a certain rule, then $t^{\prime}$ contains a redex of the same rule.

We skip the proof of the following theorem. The proof is straightforward.

3.4.2. THEOREM. (i) [Der89b] The limit of a fair, converging reduction is an w-normal form.

(ii) The limit of a fair, strongly converging reduction is a normal form.

3.4.3. COROLLARY. A reduction sequence is fair, strongly convergent if and only if it is stable.

\section{THE INFINITE CHURCH-ROSSER PROPERTY}

\subsection{Failure of the infinite Church-Rosser Property for orthogonal TRSs}

In the standard theory of orthogonal TRSs one proves the finite Church-Rosser Property after establishing the Finite Parallel Moves Lemma. The following counterexample shows that, despite the Transfinite Parallel Moves Lemma, the infinite Church Rosser property

$$
\leftarrow \omega^{\circ} \omega \rightarrow \subseteq \leq \omega \rightarrow \circ \leftarrow \leq \omega
$$

does not hold for strongly converging reductions.

\subsubsection{COUNTEREXAMPLE.}

Rules: $\quad \mathrm{A}(\mathrm{x}) \rightarrow \mathrm{x}, \mathrm{B}(\mathrm{x}) \rightarrow \mathrm{x}, \mathrm{C} \rightarrow \mathrm{A}(\mathrm{B}(\mathrm{C}))$

Sequences: $\quad C \rightarrow A(B(C)) \rightarrow A(C) \rightarrow A(A(B(C))) \rightarrow A(A(C)) \rightarrow \omega A^{\omega}$

$$
\mathrm{C} \rightarrow \mathrm{A}(\mathrm{B}(\mathrm{C})) \rightarrow \mathrm{B}(\mathrm{C}) \rightarrow \mathrm{B}(\mathrm{A}(\mathrm{B}(\mathrm{C}))) \rightarrow \mathrm{B}(\mathrm{B}(\mathrm{C})) \rightarrow \omega \mathrm{B}^{\omega}
$$

Hence $C \rightarrow \leq \omega A^{\omega}$ as well as $C \rightarrow \leq \omega B^{\omega}$. But there is no term $t$ such that $A^{\omega} \rightarrow \leq \omega t \leftarrow \leq \omega B^{\omega}$ be it converging or strongly converging.

\subsection{Böhm trees}

The counterexample and Theorem 4.1 suggest that terms having $\omega$-normal forms that are not normal forms are blocking a proof of the Infinitary Church-Rosser Property for converging reductions. From Lambda Calculus (cf. [Bar84]) we will borrow the notion head normal form (hnf), for terms that cannot be reduced to a redex and the idea for a reduction relation $\rightarrow \perp$ extending $\rightarrow$ with an extra rule: $t \rightarrow \perp$ if thas no hnf. $\perp$ is a fresh symbol that we add to the signature of the TRS.

4.2.1. DEFINITION. A term is a head normal form (hnf) if the term cannot be reduced to a redex, and a term has $a$ hnf if it can be reduced to a hnf.

4.2.2. DEFINITION. (i) Let us denote by $\perp \rightarrow$ the rewrite relation $\{\langle\mathrm{C}[\mathrm{t}], \mathrm{C}[\perp]\rangle \mid$ t has no head normal form for $\rightarrow, C[]$ is a one-place context $\}$.

(ii) Let the rewrite relation underlying Böhm reduction (notation $\rightarrow_{\perp}$ ) be $\rightarrow \cup \perp \rightarrow$. 
(iii) A term $t$ has a Böhm tree if there exists a strongly converging Böhm reduction from $t$ to $\rightarrow \perp$-normal form.

(iv) Let strict Böhm reduction (notation $\rightarrow[\perp]$ ) be the subreduction of $\rightarrow_{\perp}$ in which $\perp_{\rightarrow-}$ reduction has priority over $\rightarrow$-reduction.

We skip the proof of the following lemma and theorem.

4.2.3. LEMMA. (i) $\perp \rightarrow$ is finitely $C R$.

(ii) $\rightarrow_{\perp}$ is finitely $C R$.

(iii) Each finite part of a Böhm tree can be found in finitely many steps.

(iv) A term has at most one Böhm tree.

(v) Böhm reduction $\rightarrow_{\perp}$ and strict Böhm reduction $\rightarrow_{\perp}$ have the same normal forms.

(vi) $\rightarrow[\perp]$-reductions are strongly convergent and of lenght not more than $\omega$

(vii) Every term has a normal form with respect to $\rightarrow[\perp]$.

4.2.4. THEOREM. For both strongly convergent $\rightarrow_{\perp}$-reduction and convergent $\rightarrow_{\perp}$-reduction the infinite Church-Rosser Property holds.

\subsection{Non-unifiable orthogonal TRSs have the infinite Church-Rosser Property}

From the work of Dershowitz, Plaisted and Kaplan on convergent reductions it follows that any left-linear, top-terminating and semi- $\omega$-confluent TRS satisfies the infinite Church-Rosser property:

$$
{ }_{\omega}^{c} \leftarrow \circ \rightarrow{ }_{\omega}^{c} \subseteq \rightarrow_{\leq \omega}^{c} \stackrel{c}{c} \leq \omega \leftarrow
$$

(cf. [Der90b]: combine Theorem 1, Proposition 2 with Theorem 9.). A TRS is top-terminating if there are no top-terminating reductions of length $\omega$, that is reductions with infinitely many rewrites at the root of the initial term of the reduction. Semi- $\omega$-confluency, that is

$$
* \leftarrow \circ \rightarrow_{\omega}^{c} \subseteq \rightarrow_{\leq \omega}^{c} \stackrel{c}{c} \leftarrow
$$

holds if the Transfinite Parallel Moves Lemma holds for converging reductions. On the assumption that we are in a orthogonal TRS in which all convergent reductions are strong the infinite Church-Rosser Property holds for this TRS. Top-termination implies this assumption. Hence in top-terminating orthogonal TRSs the infinite Church-Rosser Property holds.

Using our techniques we can explain and improve this result.

4.3.1. DEFINITION. A TRS is called unifiable if the TRS contains a unifiable rule, that is a rule $l \rightarrow \mathrm{r}$ such that for some substitution $\sigma$ with finite and infinite terms for variables $1 \sigma=\mathrm{r}^{\sigma}$.

Note that unifiability in the space of finite and infinite terms means unifiability "without the occurs check": the terms $I(x)$ and $x$ are unifiable in this setting, and their most general unifier is the infinite term $I^{\omega}$. Collapsing rules, i.e. rules which right-hand side is a variable are unifiable.

4.3.2. LEMMA. The following are equivalent for an orthogonal TRS:

(i) the TRS is non-unifiable,

(ii) all convergent reductions of the TRS are strong, 
(iii) all convergent reductions are top-terminating.

4.3.3. THEOREM. Any non-unifiable orthogonal TRS has the infinite Church-Rosser Property for converging reductions.

The theorem follows from the quoted results of Dershowitz, Kaplan and Plaisted. Space prevents us to explain another proof: Non-unifiable TRSs are a special instance of noncollapsing TRSs, i.e. TRSs in which there are no rules whose right-hand side is a single variable. In non-collapsing orthogonal TRSs the infinite Church-Rosser Property holds for strongly converging reductions (cf. [Ken90a,b]). In fact we may admit one single collapsing rule of the form $I(x) \rightarrow x$ and still retain the infinite Church-Rosser property (cf. [Ken90a,b]). Note, for instance, that the collapsing rule for $\mathrm{K}$ in Combinatory Logic disturbs the infinite Church-Rosser property. A counterexample is not difficult to construct.

\section{REFERENCES}

[Am80] A. ARNOLD and M. NIVAT, The metric space of infinite trees. Algebraic and topological properties, Fundamenta Informatica, 4 (1980) 445-76.

[Bar84] H.P. BARENDREGT, The Lambda Calculus, its Syntax and Semantics, 2nd ed., (North-Holland, 1984).

[Bar87] H.P. BARENDREGT, M.C.J.D. VAN EEKELEN, J.R.W. GLAUERT, J.R. KENNAWAY, M.J. PLASMEIJER, and M.R. SLEEP, Term graph rewriting, Proc. PARLE Conference vol II, LNCS vol. 259, pp. 141-158 (Springer-Vcrlag, 1987).

[Der89a] N. DERSHOWITZ and S. KAPLAN, Rewrite, rewrite, rewrite, rewrite, rewrite, Principles of programming languages, Austin, Texas, 1989, pp. 250-259.

[Der89b] N. DERSHOWITZ, S. KAPLAN and D.A. PLAISTED, Infinite Normal Forms (plus corrigendum), ICALP 1989, pp. 249-262.

[Der90a] N. DERSHOWITZ and J.P. JOUANNAUD, Rewrite Systems, to appear in Handbook of Theoretical Computer Science (cd. J. van Lceuwen) vol.B, chapter 15, North-Holland.

[Der90b] N. DERSHOWTTZ, S. KAPLAN and D.A. PLAISTED, Rewrite, rewrite, rewrite, rewrite, rewrite to appear.

[Far89] W.M. FARMER and R.J. WATRO, Redex capturing in term graph rewriting, in Computing with the Curry Chip (eds. W.M. Farmer, J.D. Ramsdell and R.J. Watro), Report M89-59, MITRE, 1989.

[Hud88] P. HUDAK et al, Report on the Functional Programming Language Haskell. Draft Proposed Standard, 1988.

[Hue79] G. HUET and J.-J. LÉVY, Call by need computations in non-ambiguous linear term rewriting systems, Report 359, INRIA, 1979.

[Kel55] J.I. KELLEY, General Topology, Graduate Texts in Mathematics 27, Springer-Verlag New York, 1955, Second Printing 1985.

[Ken90a] J.R.KENNAWAY, J.W. KLOP, M.R. SLEEP and F.J. DE VRIES, Transfinite reductions in orthogonal term rewriting systems (Full paper), report CS-R9041, CWI, Amsterdam, 1990.

[Ken90b] J.R.KENNAWAY, J.W. KLOP, M.R. SLEEP and F.J. DE VRIES, An infinitary Church-Rosser property for non-collapsing orthogonal term rewriting systems, report CS-R9043, CWI, Amsterdam, 1990.

[Klo80] J.W. KLOP, Combinatory reduction systems, Mathematical Centre Tracts no. 127, CWI, Amsterdam, 1980.

[Klo91] J.W. KLOP, Term rewriting systems, to appear in Handbook of Logic in Computer Science, Vol I (eds. S. Abramsky, D. Gabbay and T. Maibaum), Oxford University Press, 1991.

[Pey87] S.L. PEYTON JONES, The Implementation of Functional Programming Languages, (Prentice-Hall, 1987).

[Ros73] B.K. ROSEN, Tree manipulating systems and Church Rosser theorems, JACM 20 (1973) 160-187.

[Tur85] D.A. TURNER, Miranda: a non-strict functional language with polymorphic types, in J.-P. Jouannaud (ed.), Proc. ACM Conf. on Functional Programming Languages and Computer Architecture, Lecture Notes in Computer Science, vol. 201, Springer-Verlag, 1985. 